\title{
Mössbauer Studies of Fe-doped La-Ca-Mn-O Colossal Magnetoresistive Perovskites
}

\author{
Zhao-hua Cheng $^{* \text {,** }}$, Zhi-hong Wang*, Nai-li Di ${ }^{*}$, Rui-wei Li*, R.A. Dunlap ${ }^{* *}$, and Bao-gen Shen ${ }^{*}$ \\ "State Key Laboratory of Magnetism, Institute of Physics, Chinese Academy of Sciences, Beijing \\ 100080, P.R. China \\ ***Department of Physics, Dalhousie University, Halifax, Nova Scotia, Canada, B3H 3J5
}

The discovery of colossal magnetoresistance (CMR) in the manganite perovskites $\mathrm{La}_{1-\mathrm{x}} \mathrm{Ca}_{\mathrm{x}} \mathrm{MnO}_{3}$ has recently attracted significant scientific attention[1,2]. A local structural distortion of $\mathrm{MnO}_{6}$ octahedron resulting from the $\mathrm{Mn}^{3+} \mathrm{Jahn}$-Teller effect was assumed to play an important role in determining the resistivity behavior and the magnetic transition temperature[3]. Since the longrange structural distortion decreases rapidly in $\mathrm{La}_{1-\mathrm{x}} \mathrm{Ca}_{\mathrm{x}} \mathrm{MnO}_{3}$ compounds with increasing $\mathrm{Ca}$ concentration, a local structural probe is required to analyze the distortion of the $\mathrm{MnO}_{6}$ octahedron. Mössbauer spectroscopy is a powerful technique for investigating the hyperfine interactions. One of the hyperfine parameters, the quadrupole splitting $(\Delta)$ is very sensitive to the distribution of surrounding electrons of resonant nuclei. A few percent of $\mathrm{Fe}$, which substitutes for $\mathrm{Mn}$ in $\mathrm{La}_{1-}$ ${ }_{\mathrm{x}} \mathrm{Ca}_{\mathrm{x}} \mathrm{MnO}_{3}$ compounds, can be used as a micro-probe to detect the symmetry of the nearest-neighbor $\mathrm{O}^{2-}$ ions in the $\mathrm{Mn}(\mathrm{Fe}) \mathrm{O}_{6}$ octahedron. Since $\mathrm{Mn}^{3+}$ ions are mainly replaced by $\mathrm{Fe}^{3+}$ ions in this $\mathrm{Fe}-$ doping range and both ions have identical ionic radii in six-fold octahedral coordination [4], the substitution of $\mathrm{Fe}^{3+}$ for $\mathrm{Mn}^{3+}$ does not change the tolerance factor, and consequently, the Jahn-Teller effect can be investigated separately. In this work, we summarized the application of Mössbauer spectroscopy in La-Ca-Mn-O CMR pervoskites. The local structural distortion and Jahn-Teller coupling have been determined by Mössbauer spectroscopy on the basis of quadrupole splitting. Furthermore, the connection between Jahn-Teller coupling and CMR effect has been discussed.

Rietveld profile refinement of x-ray diffraction (XRD) patterns reveals that all samples are singlephase with an orthorhombically distorted perovskite structure (Pbnm space group). The difference of $\mathrm{Mn}(\mathrm{Fe})-\mathrm{O}$ bond lengths in $\mathrm{Ca}$-doped perovskites cannot be detected within the experimental error, implying that the long-range structural distortion rapidly decreases and becomes indistinguishable by XRD technique. However, a theoretical model has predicted that strong local distortions of $\mathrm{MnO}_{6}$ octahedra would persist over a wide range of compositions in the $\mathrm{T}>\mathrm{T}_{\mathrm{c}}$ insulating phase[5].

In order to verify this assumption, Mössbauer spectroscopy utilizes ${ }^{57} \mathrm{Fe}$ nuclei as a local microprobe to determine the structural information about the $\mathrm{Mn}(\mathrm{Fe}) \mathrm{O}_{6}$ octahedra. ${ }^{57} \mathrm{Fe}$ Mössbauer spectra not only clearly indicate the presence of quadrupole splitting but also show the occurrence of a second quadrupole split doublet. These two doublets are related to the contributions of the high-spin $\mathrm{Fe}^{3+}$ and low-spin $\mathrm{Fe}^{4+}$, respectively. The quadrupole splitting for both $\mathrm{Fe}^{3+}$ and $\mathrm{Fe}^{4+}$ ions confirms the local distortion of the $\mathrm{Mn}(\mathrm{Fe}) \mathrm{O}_{6}$ octahedron. These results demonstrate that Mössbauer effect is very sensitive to the local structural information.

According to the relationship between the second-order crystalline electrical field coefficient, which obtained from Mössbauer results, as well as the Jahn-Teller coupling, the value of Jahn-Teller coupling were determined. With increasing $\mathrm{Ca}$ concentration, the Jahn-Teller coupling energy 
decreases from about $1.4 \mathrm{eV}$ for $\mathrm{x}=0$ to about $0.83 \mathrm{eV}$ for $\mathrm{x}=0.27 \sim 0.39$. The transport properties of these perovskites are governed by the interplay of the doubleexchange coupling between $\mathrm{Mn}^{3+}$ and $\mathrm{Mn}^{4+}$ ions as well as the Jahn-Teller coupling. For the samples with $\mathrm{x}=0.27 \sim 0.39$, the relatively weak Jahn-Teller coupling delocalizes the electrons and makes the hoping process become easier via double-exchange coupling below $\mathrm{T}_{\mathrm{c}}$. Therefore, these samples show a ferromagnetic metals state at $\mathrm{T}<\mathrm{T}_{\mathrm{c}}$. At $\mathrm{T}>\mathrm{T}_{\mathrm{c}}$, disorders in the spins result in a decrease of double-exchange coupling, and the JahnTeller coupling plays an important role in determining electrical resistance. Therefore, a transition from ferromagnetic metal to paramagnetic insulator can be found near Curie temperature. An applied magnetic field aligns the spins, which increases the kinetic energy, and hence decreases the effective electron-phonon coupling, leading to a large change in resistance. With further increasing $\mathrm{Ca}$ concentration, Mössbauer results indicate that the Jahn-Teller coupling becomes stronger again. The larger energy separation between $d_{z} 2$ and $d_{x^{2}-y^{2}}$

tends to trap electrons in the low-lying energy level orbitals, and hence localize the conduction electrons. Therefore, for the samples with $x>0.5$, the ground state becomes insulating and antiferromagnetic again.

Our work not only confirms the local structural distortion of $\mathrm{MnO}_{6}$ octahedron, but also reveals that Mössbauer spectroscopy can be effectively employed to investigate the Jahn-Teller effect in coupling in $\mathrm{La}_{1-\mathrm{x}} \mathrm{Ca}_{\mathrm{x}} \mathrm{MnO}_{3}$ perovskites.

\section{References}

[1]S. Jinet al., Science, 264(1994) 413.

[2]P.Schiffer et al., Phys. Rev. Lett. 75(1995) 3336.

[3]A.J. Millis et al, Phys. Rev. Lett. 74(1995)5144.

[4]J.B.A.A. Elemans et al., J. Solid State. Chem. 3(1971) 238.

[5]A.J. Millis, Nature, 392(1998) 147.
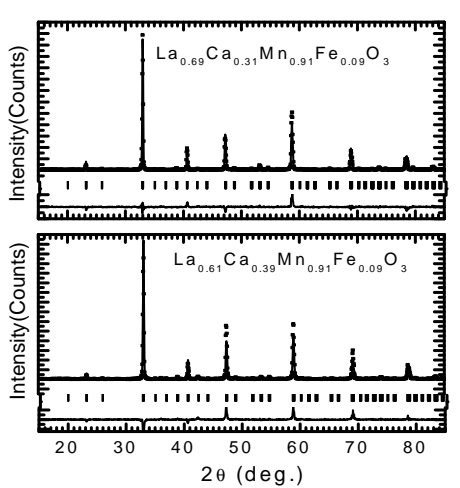

Fig.1. XRD patterns of $\mathrm{La}_{1-\mathrm{x}} \mathrm{Ca}_{\mathrm{x}} \mathrm{Mn}_{0.91} \mathrm{Fe}_{0.09} \mathrm{O}_{3}$.

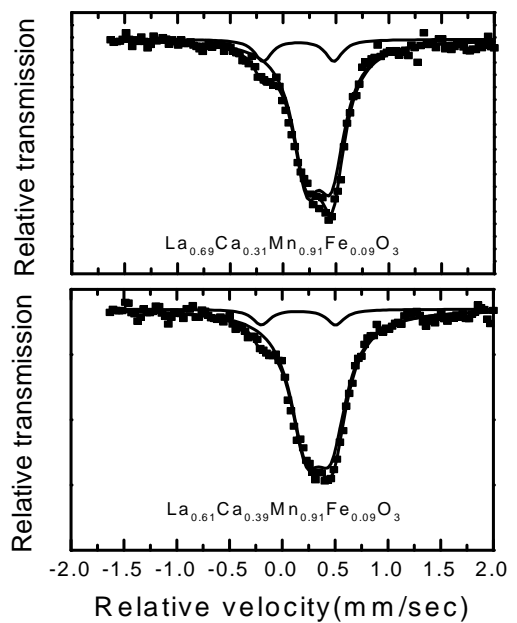

Fig.2. Mössbauer spectra of $\mathrm{La}_{1-\mathrm{x}} \mathrm{Ca}_{\mathrm{x}} \mathrm{Mn}_{0.91} \mathrm{Fe}_{0.09} \mathrm{O}_{3}$.

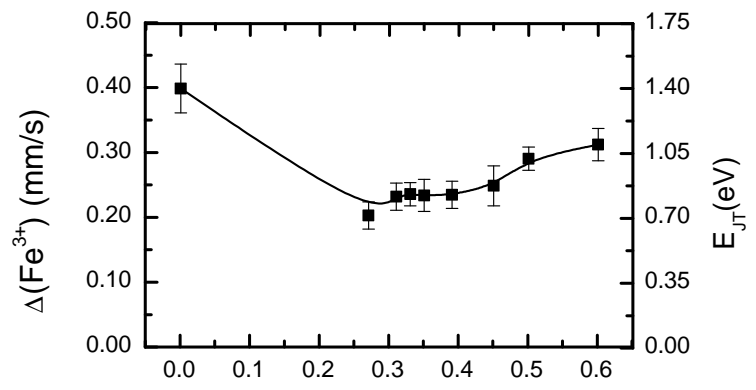

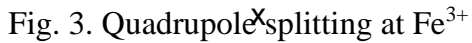
ion of $\mathrm{La}_{1-\mathrm{x}} \mathrm{Ca}_{\mathrm{x}} \mathrm{Mn}_{0.91} \mathrm{Fe}_{0.09} \mathrm{O}_{3}$ perovskites at room temperature. 